\title{
Interculturalidad en educación superior: experiencia en educación inicial en La Araucanía, Chile
}

\author{
Interculturality in higher education: experience in \\ preschool education in La Araucanía, Chile \\ Soledad Morales-Saavedra / smorales@uct.cl \\ https://orcid.org/0000-0001-8216-118X \\ Segundo Quintriqueo-Millán / squintri@uct.cl \\ https://orcid.org/0000-0002-7228-4095 \\ Pilar Andrea Uribe-Sepúlveda / pilar.uribe@uautonoma.cl \\ https://orcid.org/0000-0001-9166-9081 \\ Katerin Arias-Ortega / ariaskaterin@gmail.com \\ https://orcid.org/0000-0001-8099-0670 \\ Universidad Católica de Temuco, Chile
}

\begin{abstract}
The objective of the article is to present results of the experience developed in the processes of initial teacher training. The investigation maintains that in the indigenous Mapuche families and communities present in Chile, there is knowledge associated with socio-cultural practices, which would allow supporting a Nursery Education, from an intercultural educational approach, with an epistemic basis in the social memory of parents and sages. The main results reveal key themes in the training, regarding knowledge for intercultural education for children under six years, strengthening communication and work with family and community.
\end{abstract}

Key words: intercultural, intercultural educational approach, initial teacher training, education and childhood, Mapuche educational knowledge.

Resumen: El objetivo del artículo es presentar resultados de la experiencia desarrollada en los procesos de formación inicial docente. La investigación sostiene como supuesto que en las familias y comunidades indígenas mapuches presentes en Chile existen conocimientos asociados a prácticas socioculturales, que permitirían sustentar una Educación Parvularia, desde un enfoque educativo intercultural, con base epistémica en la memoria social de padres y sabios. Los principales resultados develan temas claves en la formación, respecto a los saberes para una educación intercultural para niños menores de seis años, fortaleciendo la comunicación y trabajo con la familia y la comunidad.

Palabras clave: interculturalidad, enfoque educativo intercultural, formación inicial docente, educación e infancia, conocimientos educativos mapuches. 


\section{Introducción $^{1}$}

La Carrera de Educación Parvularia ${ }^{2}$ de la Universidad Católica de Temuco, en Chile, se ha especializado en dos menciones: Educación Intercultural, y Pedagogía y vinculación familiar-comunitaria. La primera se focalizó en la construcción de un espacio de diálogo entre los saberes y conocimientos indígenas y occidentales. La segunda, en la participación de la familia en el proceso educativo, el reconocimiento de la escuela hacia la familia como institución social y la valoración de los distintos modos de vida, los cuales son la base fundamental para generar condiciones óptimas de desarrollo y aprendizaje de los niños.

La primera mención se basa en la declaración de las Naciones Unidas (2008: 3) sobre los derechos de los pueblos indígenas, donde se sostiene "el derecho de las familias y comunidades indígenas a seguir compartiendo la responsabilidad por la crianza, la formación, la educación y bienestar de sus hijos en consonancia con los derechos del niño". En el contexto internacional, la Convención sobre los Derechos del Niño (1989), en concordancia con el Convenio 169 de la Organización Internacional del Trabajo (OIT, 1989) y la Declaración de las Naciones Unidas sobre los derechos de los pueblos indígenas (Naciones Unidas, 2008), exigen el respeto y garantía de la diversidad cultural, que en el ámbito educativo cobra especial interés.

La incorporación del enfoque de interculturalidad es apoyado por resultados de investigaciones en la línea de educación e interculturalidad -proyectos: núm. 11075083, 2007-2009, núm. 1110489, 2011-2013 y núm. 1140490, 2014-2017, financiados por el Fondecyt-. Dichos proyectos están referidos al estudio sobre los conocimientos mapuches como base para la formulación de contenidos educativos interculturales. En el marco de estas investigaciones se han constatado categorías de contenidos educativos como identidad, patrones de socialización, valores educativos, conocimiento sobre la familia, sobre el territorio y la naturaleza, enmarcados en una perspectiva de derechos propios.

1 Este artículo de investigación científica se inscribe en las Ciencias Sociales y Humanas, Cuestiones Étnicas y Educación, y fue financiado por el Fondo Nacional de Desarrollo Científico y Tecnológico (Fondecyt) Regular núm. 1140490 para su realización.

2 En Chile se denomina Educación Parvularia a la educación de niños menores de seis años. El profesional a cargo se denomina educador de párvulos (equivale a educador de infancia o educador inicial). 
No obstante, históricamente, la escolarización de niños y niñas indígenas se realiza sobre la base de contenidos y finalidades educativas monoculturales occidentales que se contraponen al conocimiento indígena (Quintriqueo, 2010). Así, el conocimiento occidental refiere a categorías del saber disciplinar consignado en los ámbitos y núcleos de aprendizaje del currículum escolar; mientras que el conocimiento indígena refiere a un conjunto de saberes representados en la memoria individual y social de la familia y la comunidad (Quintriqueo, 2010). Según Quintriqueo, es en el contexto de la familia donde los niños aprenden un conjunto de saberes y conocimientos respecto al medio natural, social y cultural, que les permiten desenvolverse correctamente en la comunidad.

El contexto de modernidad y complejidad que presenta la población indígena en el medio escolar da cuenta de un conflicto sociocognitivo e identitario que dificulta su capacidad de desenvolvimiento, tanto en la comunidad como en la sociedad nacional. Es así como "la crisis de identidad resulta de conflictos biográficos vinculados con procesos sociales e históricos” (Quintriqueo, 2010: 13), donde los niños que se incorporan a la educación escolar adquieren un conjunto de contenidos y finalidades educativas, objetivos de enseñanza y aprendizaje, que se fundamentan en lógicas de saberes y conocimientos educativos occidentales.

Sobre lo anterior, la investigación de Carbonell (2001) señala que muchos niños indígenas en su primer contacto con la escuela sufren un triple trauma: psicológico, lingüístico y cultural. En el aspecto psicológico, enfrentan estereotipos de inferioridad, basados en el desprecio de los profesores hacia su lengua materna, hábitos y valores diferentes, que generan en el niño temor e inseguridad (Carbonell, 2001). En el aspecto lingüístico, al ingresar a la edad escolar el niño ya ha estructurado su lengua, que en general es negada junto con su cultura, deseando liberarse de la estigmatización que significa (Carbonell, 2001). En el aspecto cultural, constituye un contenido central de la socialización, que al ser negado en la educación escolar afecta en una pérdida progresiva de su identidad sociocultural (Carbonell, 2001). El conjunto de dichos factores son de vital importancia para el desarrollo de una educación intercultural en el contexto mapuche.

En este marco, el problema que moviliza esta experiencia intercultural en la formación de educadores de párvulos es la ausencia de conocimientos propios de la población mapuche en los actuales programas de formación inicial de educadores de párvulos. Esto se traduce en la invisibilización de los niños indígenas y la consecuente desigualdad en la educación de niños menores de seis años. Dicho problema está asociado a un proceso de formación inicial 
docente que históricamente se ha desarrollado con base en un programa de estudio disciplinario, homogeneizante y monocultural (Quintriqueo, 2010). Así, la formación inicial docente y la Educación Parvularia traen como consecuencia una homogeneización del trabajo educativo, la sobre-escolarización y pérdida de los sentidos esenciales de la Educación Parvularia (Peralta, 2012).

Mediante estas experiencias nos propusimos develar los conocimientos educativos deseables de incluir en una formación del educador de párvulos, que recoja la perspectiva del enfoque intercultural. La incorporación de este en la formación de pregrado, se respalda en los nuevos estándares orientadores del educador de párvulos, donde se consigna la necesidad de enseñar a sus estudiantes el respeto hacia los demás, sobre la base de la igualdad de derechos de todas las personas, valorando su diversidad sociocultural (Mineduc, 2012).

En esta lógica, la propuesta de mención de interculturalidad en la formación de estudiantes de Educación Parvularia permitirá incorporar los conocimientos educativos indígenas que son deseables de considerar en una formación de pregrado de Educación Parvularia, con el aporte de sabios, padres y madres. Lo anterior, posibilitará construir el desarrollo de competencias profesionales en el educador de párvulos que se desempeña en contextos interculturales, para potenciar y fortalecer en la formación la capacidad de conocer, comprender, respetar y valorar las diferencias sociales y culturales. Finalmente, nuestro objetivo es comunicar los resultados de una experiencia de formación inicial docente en Educación Parvularia, la cual, sustentada en un enfoque educativo intercultural, aspira a robustecer los procesos de enseñanza fortaleciendo la comunicación y el trabajo con la familia y la comunidad.

\section{Enfoque educativo intercultural}

El enfoque educativo intercultural busca mejorar la calidad de la enseñanza y aprendizaje en relación con el saber indígena-occidental y viceversa, como un aprendizaje mutuo, para generar una universalidad de saberes y conocimientos, fruto del diálogo intercultural (Quintriqueo et al., 2014). Este diálogo tiene como propósito la relativización de las categorías culturales propias y el universo simbólico, con el fin de incorporar los aportes de las otras culturas al desarrollo individual y social de los sujetos (Ferrão, 2010; Quintriqueo, 2010; Abdallah-Pretceille, 2011).

Mediante este enfoque se promueve una educación que valora y respeta las diferencias, asumiéndola como una oportunidad para enriquecer 
los procesos de enseñanza-aprendizaje. Este proceso nos permite constituir sistemas de relaciones más humanas, comprensivas, capaces de reconocer las diferencias, ventajas y desventajas, configurando un paradigma alternativo de interculturalidad crítica (Tubino, 2005b y 2011; Viaña et al., 2010). Además, dicho enfoque posibilita el estudio y comprensión de personas pertenecientes a esferas sociales y culturales distintas, lo cual relativiza de alguna manera los conocimientos y obliga a construir un saber multipolarizado, para superar la discriminación y el racismo (Ouellet, 2002; Schmelkes, 2009 y 2012; Quintriqueo, 2010; Essomba, 2012).

En el contexto de comunidades indígenas, la educación basada en un enfoque de interculturalidad significa ir más allá de los límites del Estado neoliberal, moderno y mercantilizado; es decir, significa pensar la formación profesional para la autonomía epistémica, política, social, cultural, económica y territorial, desde un pluralismo epistemológico, donde se requiere de una ciudadanía más justa, democrática e intercultural (Ouellet, 2002; Akkari, 2009; Tubino, 2011; Quintriqueo et al., 2014). Así, la educación desde este enfoque se convierte en una alternativa que permite reconocer la diversidad de saberes y conocimientos que convergen en la escuela.

En esta lógica, los contextos socioculturales y naturales constituyen el punto de partida y espacios educativos relevantes, para el aprendizaje del patrimonio cultural propio en los educandos (Freire, 2002; Akkari, 2009; Tubino, 2014; Quintriqueo et al., 2015). En consecuencia, el enfoque educativo intercultural se constituye en una aproximación teórica, práctica y metodológica que permite estudiar y comprender las formas de relacionarse entre sujetos que pertenecen a culturas y sociedades diversas. En este proceso, las formas y modos de concebir el mundo, asociado al pensar, hablar, escuchar, solucionar problemas y actuar en determinadas situaciones, valorarse a sí mismo y al otro como un legítimo otro, en cuanto individuo y grupo social, conforman aspectos centrales (Tubino y Ardito, 1996).

Es un enfoque que toma como base la episteme de las culturas y sociedades en un contexto sociohistórico y territorial determinado, en dos dimensiones: 1) una relación simétrica donde se genera un diálogo de poder entre los grupos y colectividades socioculturales, para la toma de decisiones y de reconocimiento recíproco, y 2) reconocimiento de los respectivos marcos epistémicos de los saberes y conocimientos educativos. Esta última dimensión es la base para desarrollar una educación intercultural, valorando las diferencias sociales y culturales como riqueza para la formación de futuros ciudadanos, progresivamente más interculturales. 


\section{Presentación de experiencia}

La experiencia se desarrolla en la Universidad Católica de Temuco, la cual se ha caracterizado por basarse en un plan curricular sustentado en la monoculturalidad eurocéntrica, donde el perfil de egreso no considera competencias para desempeñarse en contextos indígenas e interculturales. En la búsqueda de responder mejor a las características de los estudiantes y a las necesidades del entorno, la carrera decidió brindar a sus alumnos la oportunidad de especializarse en educación intercultural, a través de la mención en Educación Intercultural, desde el año 2011.

En estos cinco años, la mención ha especializado a 57 estudiantes y ofrece las siguientes asignaturas: 1) Programas y experiencias educativas en infancia e interculturalidad, 2) Valores y saberes en educación intercultural, 3) Diálogo como encuentro con el otro, y 4) Desarrollo de competencias en contextos interculturales. Además, incluye dos asignaturas de práctica pedagógica denominadas: a) Taller Pedagógico V, el cual tiene como ejes centrales los programas y experiencias educativas en infancia e interculturalidad, y valores y saberes en educación intercultural; y b) Taller Pedagógico VI, el cual tiene como ejes centrales el desarrollo de competencias interculturales y el diálogo como encuentro con el otro.

Dichas prácticas pedagógicas se desarrollan en aulas educativas, con presencia de niños mapuche y no mapuche, tanto en el medio urbano como rural. De esta manera, mediante la mención de interculturalidad nos proponemos formar educadores que favorezcan la construcción de un espacio de diálogo entre conocimientos educativos indígenas y occidentales, posibilitando al niño y niña mantener y fortalecer su propia cultura. Además, pretendemos desarrollar en las estudiantes competencias que les permitan vincular la escuela con la familia y la comunidad, como agentes educativos.

El objetivo general es fortalecer la interculturalidad en la formación de inicial docente. Dentro de los objetivos específicos se señalan: fortalecer la atención y educación de calidad en contextos de diversidad étnica y cultural, a través de la comprensión y mejoramiento de sus concepciones y prácticas pedagógicas acerca de posibilidades y limitaciones para incorporar contenidos educativos mapuches al currículo formativo, desde un enfoque educativo intercultural; sensibilizar a los futuros profesionales para valorar y respetar las diferentes culturas y promover el aprecio, respeto y sana convivencia; fortalecer el resguardo de los derechos de todos los niños, en especial de quienes se encuentran en una situación de desigualdad por su pertenencia étnica; incorporar contenidos educativos mapuches, para contextualizar la 
enseñanza en contextos interculturales; diseñar, aplicar y evaluar un proyecto educativo intercultural, para potenciar la interculturalidad en aulas infantiles, integrando a las familias y comunidades; y lograr la equidad e igualdad entre la población indígena y no indígena, promoviendo el resguardo sobre los derechos del niño.

\section{Resultados del proceso}

Los resultados de la experiencia educativa se sustentan en tres categorías: a) Conocimientos y saberes educativos mapuche, b) Desarrollo de competencias formativas y c) Fortalezas y debilidades en el proceso formativo.

\section{a) Conocimientos y saberes educativos mapuches}

Esta categoría se construye a partir de las interacciones de las estudiantes de la carrera con los kimches (sabios mapuches), padres, madres y familias de comunidades mapuches, en el marco de sus prácticas pedagógicas en las instituciones educativas. En este proceso, las estudiantes realizan entrevistas, en relación con saberes y conocimientos educativos mapuches para la formación de los niños y niñas. La información recogida permitió identificar cuatro categorías centrales de saberes educativos: 1) Respeto de la familia, 2) Persona integral, 3) Normas de convivencia y 4) Relación con la naturaleza.

Respecto a la categoría Respeto de la familia, se ha identificado que los niños y niñas son socializados desde su primera infancia a partir de interacciones, donde aprenden e interiorizan "elementos socioculturales de su medio ambiente, los integran en la estructura de su personalidad, bajo la influencia de experiencias y de agentes sociales significativos, adaptándose al entorno social en donde vive" (Essomba, 2014: 37).

En esta categoría se rescata de las entrevistas: El gülam (consejo), como un método de enseñanza de contenidos, procedimientos y actitudes "para enseñar a niños y adolescentes valores, normas y creencias de la familia y la comunidad" (Carihuentro, 2007: 7); este método educativo tiene como finalidad la enseñanza del yamuwün, que se refiere al respeto mutuo entre las personas. El yamuwün es una macrocategoría que involucra el respeto que nace a partir de un ser superior (günechen), que representa un grado espiritual hacia fuerzas espirituales y sobrenaturales, y también respeto hacia las personas que constituyen un rol en la comunidad; por ejemplo, la machi, quien es definida como la persona que ejerce un oficio socio-religioso, que posee y transmite los conocimientos en relación con la medicina, la salud y las fuer- 
zas espirituales de los espacios verticales y horizontales del mundo mapuche (Quintriqueo et al., 2016: 161).

Desde esta perspectiva, la familia es un grupo social encargado de entregar al niño un sentimiento de pertenencia que le permite reconocerse como hombre o mujer participante de un grupo por las características en común que tiene con los individuos que lo conforman. El valor de la familia significa expresar, respetar y quererse entre las personas, manifestándose una actitud de amor y respeto. Esto incluye la obediencia a la tradición mapuche y a las personas mayores portadoras de saberes y conocimientos; así como el cariño hacia los niños y el cuidado en el trato con adolescentes y jóvenes, para actuar en el medio familiar y comunitario. Se señala en las conversaciones con los kimches que el conocimiento adquirido históricamente por los mapuches se atribuye a fuerzas espirituales expresadas en la idea de un ser supremo que entrega la sabiduría y llevaría por el buen camino a las personas.

Asimismo, los kimches señalan que para formar a los niños en las comunidades se les debe llevar por el camino de los saberes mapuches; esto implica el trabajo con la familia y personas que tienen el conocimiento, para orientar el proceso de formación del niño. Desde la memoria social mapuche se sostiene que los padres y abuelos dialogan con los niños y niñas utilizando metáforas, moralejas, además de epew (relato o cuento), pewmas (sueños), feyentun (leyendas) y guxam (conversación), las cuales son repetidas hasta que el niño las aprenda. Lo anterior se complementa con procesos de observación e imitación de actividades cotidianas que realizan sus padres a través del juego y el trabajo.

En relación con la categoría Persona integral, esta se refiere a la formación de personas según el conocimiento educativo mapuche. Para Quilaqueo (2006), el valor de educar está dado por los kimches, quienes tienen conocimientos adquiridos por su experiencia con el medio natural, social y cultural. Así, el conocimiento se transmite de acuerdo a un proceso de formación individualizado y dependiendo del grado de saber de la persona. Este se realiza a través de la participación temprana de los niños y niñas en actividades de la vida cotidiana a nivel familiar y comunitario.

Su finalidad es que el niño aprenda observando e imitando las actividades que hacen los adultos de su entorno familiar, para orientar su conducta dentro de los parámetros valóricos y culturales mapuche; es decir, implican un crecimiento de la persona mediante la interpretación y la comprensión de normas, valores y reglas que se encuentran insertas en la sociedad. Desde la perspectiva mapuche, la formación de la persona es una acción socioeducativa denominada kimeltuwün. 
Según Quilaqueo (2006), el kimeltuwün se define como el conocimiento educativo que tiene por objetivo transmitir estructuras y significados del patrimonio cultural mapuche desde cuatro ámbitos: el intelecto, los valores, las actitudes y su capacidad física, para ser valorado en el contexto familiar y comunitario. Es así como el niño y niña que recibe su kimeltuwün debe manifestar küme kimün (buen conocimiento) y küme rakizuam (buen pensamiento).

Respecto a la categoría Normas de convivencia, se presenta como uno de los primeros conocimientos que deben adquirir los niños y corresponde a una norma cultural importante para el ámbito actitudinal en la relación con su comunidad. El respeto hacia las personas se logra cuando alguien, conscientemente, sabe la importancia del respeto a los valores y es capaz de respetar a la comunidad, a la familia, a las personas de edad y, por sobre todo, a la vida del ser humano, especialmente a quienes tienen sangre mapuche. Ejemplo de ello es el saludo, pues mediante su práctica los niños generan los primeros lazos y acercamientos con los demás miembros de la comunidad, además de adquirir las primeras normas culturales mediante el chaliwün (saludo), desde la lógica mapuche.

En cuanto a la categoría Relación con la Naturaleza, se refiere a la interacción y respeto con la naturaleza, las creencias, la lengua, el estilo de vida mapuche y a no asistir a lugares prohibidos; por ende, se traduce en una estrecha relación entre el mapuche y la mapu (tierra), y se desarrolla en los niños mediante los diferentes espacios físicos que se encuentran en el lugar que habitan. Es así como desde la lógica cultural mapuche se les enseña y transmite el respeto y la relación que debe existir con todos los elementos que forman parte de la naturaleza, con la conciencia de que las personas forman parte de dicho entorno y que deben vivir en armonía con cada uno de ellos.

Se destaca la importancia de que el niño adquiera conciencia sobre las fuerzas que están presentes en la naturaleza, así como también el respeto y cuidado con estos. Dichas enseñanzas pueden servir como advertencias para que el niño tenga mayor precaución al momento de jugar y desenvolverse en su entorno. Según Quilaqueo (2006), los conocimientos y saberes educativos mapuche pueden ser de tres tipos: conceptual, procedimental y actitudinal. El conceptual es el kimün, entendido el que permite conocer y comprender el entorno cultural (Quilaqueo, 2006).

El procedimental refiere al uso de estrategias para la transmisión de conocimientos que se utilizan para la formación de la persona y contempla el zapin, inatuzugu y gülam, los cuales están relacionados con cultivar saberes, la búsqueda del $a z$ (ser mapuche), la enseñanza de valores y actitudes de acuerdo 
al ideal del $a z$ mapuche (Quilaqueo, 2006). Por último, el conocimiento actitudinal tiene que ver con el valor de saber actuar entre las personas y abarca los tres tipos de actitudes que la persona debe manifestar respecto al saber y al conocimiento: el yamuwün se refiere al respeto o aprecio por seres y personas significativas; el azmawün es el respeto a la armonía entre el hombre y la naturaleza; y el mañummawün es el agradecimiento (Quilaqueo, 2006).

\section{b) Desarrollo de competencias formativas}

Durante el desarrollo de la mención hemos podido evidenciar ciertas competencias en nuestras estudiantes, que están referidas a: 1) cambio paradigmático, 2) planificar contextualizadamente e 3) investigación e indagación.

Respecto a la primera competencia, es importante considerar que el paradigma de la interculturalidad tiene varias acepciones y contexto. La postura que se asume en este artículo se asocia al planteamiento nacido de la construcción de un conocimiento científico emergente de la interculturalidad (Ramírez, 2001). Para comprender el cambio de paradigma que logran desarrollar las estudiantes es necesario indicar los elementos que generan esta evolución.

Por una parte, está el cambio de definición sobre el concepto de interculturalidad; por otra, el entender este concepto como un proceso de convivencia; y, finalmente, la organización y abordaje de los contenidos curriculares. En relación con el primer elemento, en el inicio de la formación las estudiantes señalan concepciones reduccionistas, sesgadas y centradas en las diferencias sobre interculturalidad. Durante el transcurso de su formación, las estudiantes logran construir una concepción más amplia, conciliadora y reconocedora del aporte del otro, en cuya base está el diálogo recíproco que entre dos o más grupos genera riqueza.

Asimismo, se rompe la creencia de que hablar de interculturalidad es hablar solamente de pueblos indígenas (Altarejos, 2006; Cortés, 2009). Para el segundo elemento, las acepciones que encontramos es considerar a la interculturalidad como un proceso de convivencia humana, basado en el respeto a la relación recíproca de valores entre varias culturas. Esta acepción apela a la valoración del bagaje ético social de varias culturas, en donde la interrelación se sustenta justamente en el respeto a estos valores (Ramírez, 2001). Y para el último elemento, la ampliación del enfoque de interculturalidad como parte de la cultura escolar se logra a partir de la organización y el abordaje de los contenidos curriculares, la articulación entre los saberes educativos propios y los contenidos del área disciplinar por enseñar, convirtiéndose en un complemento para cada programa. 
De esta manera, se busca dar respuesta a las necesidades sociales, demandas educativas del medio familiar y la generación de conocimiento para contextualizar las prácticas pedagógicas en el nivel de Educación Parvularia. Desde esta perspectiva, el enfoque educativo intercultural permitiría contrarrestar los modelos educativos de compensación, de asimilación y de segregación cultural.

Un ejemplo de cambio paradigmático de las estudiantes en relación con la interculturalidad se expresa en el siguiente testimonio:

[...] poder cambiar la mentalidad en relación a este concepto y no limitarlo sólo a los pueblos originarios, sino entre grupos de personas con distinto pensamiento religioso, político, social, sexual, cultural es evolucionar. Para ello es necesario comenzar a vivir bajo la perspectiva del respeto y el diálogo, en el cual se pueden tener cabida distintas formas de pensar, que no necesariamente cambian las mías propias, pero me enriquecen (Estudiante de la mención, año 2013).

De acuerdo al testimonio, en el proceso formativo, las estudiantes han logrado asumir la interculturalidad para todos, indígenas y no indígenas. $\mathrm{Al}$ mismo tiempo, logran las competencias para distinguir las diferencias sociales del entorno donde se desempeñan profesionalmente. Sin embargo, se constata que la estudiante, en su testimonio, expresa una negación a cambiar sus actitudes de monoculturalidad. En este sentido, el cambio de actitud hacia las diferencias sociales y culturales queda en el ámbito personal y no necesariamente incide en el ámbito profesional.

Respecto a la segunda competencia, refiere a la planificación contextualizada de los patrones culturales existentes en contextos de diversidad social y cultural. Es así como las futuras educadoras de párvulo en su proceso formativo deben ser capaces de sistematizar y explicitar los intereses de los actores sociales del medio educativo, para adaptar sus intervenciones pedagógicas, como una propuesta de educación para todos. En este sentido, se puede decir que un proceso educativo intercultural contextualizado busca propiciar en los estudiantes un bagaje de aptitudes y actitudes que les permita desenvolverse en una sociedad con diversidad cultural.

Esto es, que el estudiante en formación logre el desarrollo de un conjunto de capacidades, para, en la cotidianidad, convivir con personas culturalmente diferentes, solucionar conflictos que surjan a partir de la diversidad de valores, propiciar su enriquecimiento a partir de esta realidad, ser crítico y respetuoso de otras culturas (Cortés, 2009). Dentro de los elementos que las alumnas consideran importantes a la hora de contextualizar el currículo escolar, destaca conocer los patrones familiares: 
Conocer, planificar y considerar los elementos familiares y culturales de su comunidad, hacer que los niños valoren sus tradiciones, que no sientan vergüenza de su origen, pero siempre considerando lo que plantean los marcos curriculares que el Mineduc tiene, es importante que los niños ni la educadora pierdan eso (Estudiante de la mención, año 2014).

Las estudiantes, en su mayoría, manifiestan la importancia de considerar los saberes educativos propios en la planificación de sus prácticas pedagógica, para propiciar en los niños la valoración de su identidad cultural. Desde la perspectiva de las alumnas, dicha planificación debe permanecer en el marco de los contenidos que se establecen en el currículum escolar.

En relación con la tercera competencia, referida a la investigación e indagación del contexto educativo intercultural, las estudiantes logran identificar y diagnosticar las necesidades educativas, sociales, institucionales y personales de los actores del medio educativo. Esto tiene el propósito de promover cambios eficaces en las prácticas educativas, en la enseñanza, en la organización de los contenidos educativos, y propiciar procesos de convivencia y resolución de conflictos interculturales, lo cual se refleja en el siguiente testimonio:

En nuestra formación es importante que esté presente la investigación, especialmente referida a los contextos [educativos], lo cual facilita entender y comprender cada una de las situaciones y conflictos que se dan en el aula y permiten la atención a la diversidad (Estudiante de la mención, año 2012).

De acuerdo con el testimonio, la investigación educativa en la formación inicial docente es de vital relevancia para comprender el contexto educativo, las relaciones interculturales entre los actores del medio educativo y avanzar en la sistematización de los saberes y conocimientos propios de los estudiantes. Es así como el docente en su práctica pedagógica está llamado permanentemente a construir conocimiento desde su experiencia, mediante la investigación acción.

Un educador, además del conocimiento de la pedagogía y de su saber específico, tiene la necesidad de adquirir un saber-hacer práctico, el cual se compone de una habilidad general para la tarea educativa y de una capacidad de ajuste a las situaciones particulares que se presentan a lo largo del trabajo; es decir, la capacidad de comprender y explicar lo que sucede en la realidad educativa. Esto se logra a través de la mirada crítica que el propio educador puede hacer a su desempeño.

De acuerdo con lo anterior, Schon (1992) señala que el saber práctico puede develarse en procesos de "reflexión en la acción" o en procesos de "reflexión sobre la acción”. Para efectos de este análisis, centraremos la mirada en la última, la cual invita al educador a mirar su práctica críticamente después 
de la acción, mediante la observación y la reflexión sobre las acciones; con ello es posible entender cómo se actúa y construir modelos sobre la realidad.

Aquí es posible generar procesos investigativos bajo el modelo de la sistematización, las lecturas de la realidad, las acciones desencadenadas y el sentido que los actores educativos dan a su conducta y a la conducta de otros. Además, permite dar una explicación a las estructuras sociales donde está inmersa la práctica educativa y la forma como es afectada (Castro, 2006), aspecto que se condice con lo señalado en el siguiente testimonio:

Para finalizar, debo mencionar que este proyecto educativo intercultural nos ayudó a valorar la importancia que tiene la autocrítica y el estar en una constante reflexión de nuestro quehacer pedagógico. Esta mención es un desafío que debo llevar al aula y sobre todo con las educadoras ya tituladas, a ellas cuesta convencerlas (Estudiante de la mención, año 2013).

En síntesis, investigar desde la práctica educativa es una oportunidad para que las estudiantes logren construir un marco de referencia y un método de trabajo para analizar el quehacer pedagógico, considerando el entorno de la vida cotidiana. Esto les permitirá usar la experiencia como materia prima del trabajo intelectual y del pensamiento creativo, y desarrollar el hábito de reflexionar en y sobre la acción. Posibilita, además, recuperar las innovaciones pedagógicas, construir saber específico, y comprender la acción educativa dentro del contexto social (Castro, 2006).

\section{c) Fortalezas y debilidades del proceso formativo}

Las estudiantes reconocen en su proceso de formación dos fortalezas que se asocian a instrumentos y técnicas de recogida de información: 1) elaboración de portafolio; y 2) aprender a desarrollar un levantamiento etnográfico. Ambas fortalezas surgen de la competencia de investigación e indagación en el contexto educativo intercultural.

En relación con la elaboración del portafolio, este se constituye en un instrumento que cumple con una función formativa, al permitir monitorear y autoevaluar su desempeño para realizar la meta-cognición respecto de la evolución del aprendizaje académico en cuanto al trabajo que desarrollan en su práctica pedagógica. De esta manera, el portafolio permite la recopilación de datos de manera periódica y sistemática sobre su actuación profesional, lo cual favorece la reflexión crítica sobre la propia práctica y tomar decisiones para la mejora (Korthagen, 2001).

En cuanto a la segunda fortaleza referida al levantamiento etnográfico, las estudiantes manifiestan que esta forma de leer los fenómenos y relaciones 
que se producen en el contexto escolar les permite comprender los fenómenos de la cultura escolar en un contexto mapuche. Ello les ayuda a adaptar sus prácticas pedagógicas desde un enfoque educativo intercultural, para potenciar las relaciones sociales entre los distintos agentes del medio educativo y la comunidad.

Las debilidades que se observan en el proceso formativo se asocian a la necesidad de fortalecer en su práctica pedagógica en un contexto intercultural la comunicación y trabajo con la familia. Para eso es necesario generar instancias de trabajo en equipo con familia y miembros de la comunidad en la escuela. Es importante abordar estas debilidades, pues en el año $2002 \mathrm{el}$ Ministerio de Educación de Chile creó la política de participación de madres, padres y apoderados.

Por otro lado, ya las Bases curriculares de la Educación Parvularia (Mineduc, 2001), en sus fundamentos, señalan a la familia como primera educadora y proponen compartir con ella la misión educativa de dos grandes corrientes teóricas que disputan este campo: 1) aquella que concibe la participación de los padres como un apoyo a la eficacia del proceso educativo, $\mathrm{y}$ 2) la mirada que promueve la participación de los padres como un derecho y un espacio de ciudadanía, desde el cual todos los padres, madres y familiares son competentes y necesarios para participar en la toma de decisiones que afectan sus vidas y las de sus hijos (Navarro, 2002). El siguiente testimonio evidencia lo antes dicho:

Otra de las causas que hemos evidenciado es la escasa participación de las familias, en instancias como las reuniones de apoderados, donde estas no dan a conocer su opinión... yo le asigno un valor clave y fundamental en el proceso de formación de los niños, dada la riqueza cultural que poseen, la cual es un aporte inagotable de conocimientos y saberes tradicionales de esta cultura, los que deben transmitirse (Estudiante de la mención, 2011).

Investigaciones como la de Valverde (2008) aportan datos como el impacto positivo de la participación de los padres en la educación primaria y no así en el nivel de educación infantil; además, se indica que en este se sabe poco sobre cuáles son los aspectos más efectivos de la participación de los padres en el contexto escolar. Los resultados sugieren que la participación de los padres es un buen indicador de la calidad del servicio, pero que aún estamos lejos de evaluarla de forma efectiva (Parent Child Care Involvement, citado en Valverde, 2008).

Por su parte, Valdivia y Valverde (2006) realizaron un estudio descriptivo de la participación de los padres con hijos en jardines infantiles, evaluando la participación real, la participación deseada, y los obstáculos y facilitadores 
de ella. Las investigadoras concluyen que los resultados generales sobre lo que los padres y educadores dicen hacer respecto a la participación, permiten observar que esta se encuentra caracterizada por los tres primeros niveles planteados por Flamey et al. (1999): información, colaboración y consulta (Valdivia y Valverde, 2006).

Si bien existe un vacío en el tipo y forma de participación de los padres en la escuela, cabe preguntarse: ¿Qué sucede entonces en contextos interculturales? En función de esta debilidad, la carrera decide incorporar a la mención de interculturalidad la temática de familia y comunidad.

Dicha decisión se fundamenta en que existen vacíos acerca de cómo los diversos actores involucrados están pensando y esperando que ocurra la práctica educativa de las educadoras iniciales con las familias, durante la primera infancia. La participación de los padres se consolida como variable de calidad (Flamey et al., 1999; Santelices, 2002; Valdivia y Valverde, 2006), develando la necesidad de relacionar a la institución educativa con la familia.

\section{Conclusiones}

Las principales conclusiones de este trabajo nos permiten señalar que cuando se piensa en procesos formativos de educadores iniciales en el contexto intercultural, se deben proveer instancias académicas formales e informales que apunten a que el estudiante aprenda a entablar una relación no conflictiva con personas de otras culturas. La mayor parte de lo que se puede leer sobre los procesos educativos interculturales en educación inicial es que en escasas ocasiones se aclara cuáles son los contenidos por enseñar y las competencias que debe desarrollar un profesional de la educación al intervenir en escenarios interculturales; sólo se incide en el hecho de que sean habilidades que les permitan entablar relación con las otras culturas.

El hecho de hacer hincapié en que se trata de estrategias que acerquen a las otras culturas denota, una vez más, que implícitamente no se está considerando a los otros como seres individuales y concretos, como personas, sino que se está volviendo a establecer la distinción entre ellos y nosotros por su adscripción a un grupo, como lo señala Altarejos (2006).

En cuanto a la primera categoría, conocimiento y saberes educativos mapuches, se puede concluir que no se pretende romper con el currículo formal; sino, por el contrario: la educación intercultural es una nueva forma de tratamiento entre la vinculación con la cultura local en torno al currículo, producto de la vinculación que se intenciona en el proceso formativo de estudiantes de educación de párvulos de la mención de interculturalidad. 
Estas experiencias nos han permitido develar saberes que para el contexto educativo intercultural mapuche son necesarios de considerar en el proceso educativo: el valor a la familia, la persona integral, el saludo y la relación con la naturaleza. En este sentido, el proceso educativo se desarrolla en un marco de límites más flexibles, que admiten el tratamiento de contenidos disciplinares con los propios del medio socio-familiar. Lo que queremos destacar con esto es señalar que, cuando se piensa en contextos interculturales, un aspecto de relevancia para contextualizar el currículo es descubrir e investigar en el propio medio cuáles son los contenidos que dan sentido y significado al proceso pedagógico.

Desde este paradigma, la formación en Educación Parvularia intercultural y la educación intercultural deben buscar que los estudiantes vean en las diferencias no sólo una alternativa al modo de vida de la cultura de origen, sino también una alternativa posible de ser incorporada en la escuela (Essomba, 1999). Esto significa valorar la identidad, los contenidos y finalidades educativas de los otros para la formación de una persona; construcción de conocimientos y competencias dialógicas entre el saber mapuche y occidental en el contexto escolar (Quilaqueo et al., 2014).

En cuanto a la categoría de competencias, para la mención de interculturalidad es necesario implementar un proceso continuo de acciones concretas, pero también una constante reflexión sobre el mismo quehacer en las instituciones formadoras de profesores. En este marco, la formación del profesorado en educación intercultural en el contexto nacional requiere una formación sólida en investigación y un compromiso vocacional.

La investigación debe permitir no sólo identificar líneas de pensamiento que se sustenten en una crítica epistemológica, para superar la educación intercultural funcionalista, en contextos indígenas, y la enseñanza monocultural encubierta en la escuela, en el medio familiar y social, sino también la indagación del desempeño docente tanto en la formación inicial como en el medio escolar (Quilaqueo et al., 2014).

En tanto, para la categoría fortalezas y debilidades, concluimos que es necesario desarrollar ciertas habilidades en los procesos formativos en las estudiantes, para que superen retos y dificultades a partir de la indagación, investigación, conocimiento y reconocimiento del otro o de los otros en un ambiente plural, logrando un enriquecimiento entre las partes que convergen, adquiriendo una mayor sensibilidad para identificar y solucionar problemáticas sociales, en un marco de reconocimiento y conveniencia en la diversidad. Esto tiene el propósito de alcanzar objetivos comunes, potenciando el conocimiento disciplinar integrado a la cultura institucional. 
Es evidente que ante este horizonte social, cada vez más diverso y complejo, resulta imprescindible formar a los profesionales de la educación en un estilo pedagógico ajustado a la nueva realidad, que les capacite para afrontar los nuevos desafíos y dar respuestas satisfactorias, desde la comprensión y el respeto a las diferencias. Para conseguir esto, es fundamental considerar permanente y transversalmente el principio de contextualización sociocultural de la información.

Este principio requiere que los docentes y académicos puedan situar actividades de aprendizaje y, por consiguiente, contenidos curriculares en contextos pertinentes a partir de la realidad del estudiantado, en particular aquellos que se encuentran próximos al alumno y que guardan relación con la profesión que este aspira lograr al término del proceso formativo. El mundo intercomunicado y globalizado se ha convertido en un gran reto para la formación profesional (Ferro, 2000; CINDA, 2002).

La Educación Parvularia no está ajena a las transformaciones e innovaciones necesarias a partir de los cambios de carácter interno a los cuales se enfrenta la educación superior hoy. Entre ellos, el crecimiento de la población estudiantil en la educación superior, la heterogeneidad, segmentación de la oferta educacional y el nuevo perfil de los estudiantes (Peralta, 2000; Armengol, 2005; Burnet, 2008). Es así como ya se ha empezado a investigar a nivel nacional e internacional sobre las características y limitaciones en los programas de formación inicial docente y más específicamente en educación inicial.

Mediante este artículo invitamos a más profesionales de la educación inicial a realizar investigaciones y aportes, y a generar experiencias educativas sobre la cultura de los pueblos originarios y en especial del pueblo mapuche. Es posible reconstruir la cultura a partir de la sistematización de los discursos de las familias, kimche, longko y otras personas de la comunidad como una forma de entregar un aporte a la formación de las nuevas generaciones. Una de las mayores críticas a la formación docente es que esta no capacita para enfrentar adecuadamente la práctica docente (Veenman, 1984); sino que tiende a la reproducción endogámica (Ferry, 1990; Davini, 1995), que está desprovista de conocimientos pedagógicos y sobrecargada de saberes, tratándose más bien de una formación academicista.

Dicho postulado se sustenta en resultados de estudios efectuados por Gibaja (1994), Brockbank y McGill (2002), Carr (1996) e Imbernón (2010), donde, en general, develan que los propios maestros y maestras comparten visiones críticas sobre las insuficiencias de su preparación profesional. Mérida (2009) añade que es necesario considerar medidas de acción para revertir dichos índices, y que cualquier reflexión que aborde la formación inicial de 
los futuros docentes se debería relacionar directamente con la clarificación de la identidad de la actividad de enseñanza que han de desarrollar en su futura práctica profesional.

En definitiva, este artículo aspira e invita a repensar la formación inicial docente de educadores de párvulos en la consideración de relevar los temas de interculturalidad como aspecto necesario para mejorar la calidad educativa, no sólo en el ámbito pedagógico, sino en el dialógico, como encuentro entre lo personal, social y cultural.

El enfoque intercultural, no remitido exclusivamente a lo indígena, permite abordar la diversidad cultural como un elemento transversal al desarrollo curricular del aula, aportando en la formación de los niños desde un punto de vista valórico. En esta concepción, las personas educadoras y técnicas en Educación Parvularia cumplen una labor que se complementa con el rol de las familias como primer agente educativo, en un contexto donde pueden existir muchas diversidades, incluso tantas como niños/as que asisten a la escuela.

En consecuencia, la interculturalidad no es para algunos, es para toda la sociedad global. En este sentido, como señala Cortés (2009), la educación intercultural busca fomentar actitudes positivas ante la diversidad cultural; potenciar la convivencia y la cooperación; desarrollar habilidades comunicativas verbales y no verbales, que permitan interactuar y comunicarse efectivamente, y potenciar la igualdad de oportunidades académicas. En definitiva, a lo que aspiramos en la formación de las estudiantes de la mención es que ellas se enriquezcan a partir de la interacción con otras culturas, reconociendo la diversidad como un valor positivo y de enriquecimiento mutuo.

\section{Referencias}

Abdallah-Pretceille, Martine (2011), "La pedagogie interculturelle: entre multiculturalisme et universalisme”, en Lingvarvmarena, vol. 2, Suiza: Lingvarvmarena.

Akkari, Abdeljalil (2009), Introduction aux approches interculturelles en éducation, Suiza: Université de Genève.

Altarejos, Milagros (2006), "Críticas al enfoque de educación intercultural y consecuencias prácticas”, en Estudios sobre Educación, núm. 10, España: Servicio de Publicaciones de la Universidad de Navarra.

Armengol, Carmen (2005), "Perfil y competencias de los pedagogos de hoy", en Documento curso Pedagogía, España: REDIPE.

Brockbank, Anne y McGill, Ian (2002), Aprendizaje reflexivo en la educación superior, España: Morata. 
Soledad Morales-Saavedra, Segundo Quintriqueo-Millán, Pilar Andrea Uribe-Sepúlveda y Katerin Arias-Oretga. Interculturalidad en educación superior: experiencia en educación inicial en La Araucania, Chile

Burnett, Nicholas (2008), “What kind of UNESCO for what kind of education?”, en Hugh Gaitskell Memorial Lecture. Disponible en: http://www.nottingham.ac.uk/shared/ shared_uccer/unescopdfs/Hugh_Gaitskell_Lecture_Burnett_2008_.pdf [06 de noviembre de 2016].

Carbonell, Jaume (2001), La aventura de innovar. El cambio en la escuela, España: Morata.

Carihuentro, Sergio (2007), "Saberes mapuche que debiera incorporar la educación formal en el contexto interétnico e intercultural según sabios mapuche”, Tesis de Magister en Educación mención currículum y comunidad educativa, Chile: Universidad de Chile.

Carr, Wilfred (1996), Una teoría para la educación. Hacia una investigación educativa crítica, España: Morata.

Castro, Julia (2006), "Investigación desde la práctica educativa. Una oportunidad de conocer desde el saber-hacer”, en Viref, Biblioteca Virtual en Educación Física, Colombia: Instituto Universitario de Educación Física.

CINDA (Centro Interuniversitario de Desarrollo) (2002), "Las demandas sociales y sus efectos para la educación Superior”, en Seminario de Educación, Chile: Alfabeta Artes Gráficas.

Cortés, Claudia (2009), "La interculturalidad ante un cambio de paradigma, un reto para la Educación Superior", en Revista Fuente, núm. 1, Mexico: Universidad Autónoma de Nayarit.

Davini, Cristina (1995), La formación docente en cuestión: politica y pedagogia, Argentina: Paidós.

Essomba, Miquel (1999), Construir la Escuela Intercultural: Reflexiones y propuestas trabajar la diversidad étnica y cultural, España: Graó.

Essomba, Miquel (2012), Inmigración e interculturalidad en la ciudad. Principios, ámbitos y condiciones para una acción comunitaria intercultural en perspectiva europea, España: Graó.

Essomba, Miquel (2014), "Diversity of contexts, unity of purposes: Preparing for successful school achievement by those children and youngsters in Europe from migrant background", en Journal Citizenship Teaching y Learning, núm. 2, Inglaterra: DOI: https://doi.org/10.1386/ctl.9.2.117_1.

Ferrão, María (2010), "Educación intercultural en América Latina: Distintas concepciones y tensiones actuales", en Revista Estudios Pedagógicos, núm. 36, Chile: Universidad Austral de Chile.

Ferro, Jesús (2000), Visión de la universidad ante el siglo XXI, Colombia: Universidad del Norte.

Ferry, Gilles (1990), El trayecto de la formación. Los enseñantes entre la teoría y la práctica, México: Paidós.

Flamey, Guido et al. (1999), Los centros de padres y apoderados: Nuevos actores en el control de la gestión escolar, Chile: Centro de Investigación y Desarrollo de la Educación.

Freire, Paulo (2002), Pedagogía de la esperanza, México: Siglo XXI.

Gibaja, Regina (1994), "La imagen del rol docente”, en Gibaja, Regina y Eichelbaum de Babini, Ana [coords.], La educación en la Argentina, Argentina: Itinerarios educativos.

Imbernón, Francisco (2010), "La formación del profesorado y el desarrollo del currículum", en Gimeno, José [coord.], Saberes e incertidumbres sobre el curriculum, España: Morata.

Korthagen, Fred (2001), Linking Practice and Theory. The Pedagogy of Realistic Teacher Education, Inglaterra: Lawrance Erlbaum. 
Convergencia Revista de Ciencias Sociales, núm. 77, 2018, Universidad Autónoma del Estado de México

Mérida, Rosario (2009), "Necesidades actuales en la formación inicial de las maestras y maestros”, en Revista Electrónica Interuniversitaria de Formación del Profesorado, núm. 2, España. Disponible en: http://www.aufop.com [04 de octubre de 2016].

Mineduc (Ministerio de Educación Chile) (2001), Bases Curriculares de la Educación Parvularia, Chile: Mineduc.

Mineduc (Ministerio de Educación Chile) (2012), Estándares orientadores para carreras de Educación Parvularia. Chile: Mineduc.

Naciones Unidas (2008), Declaración de las Naciones Unidas sobre los derechos de los pueblos indigenas, Suiza: Naciones Unidas.

Navarro, Gracia (2002), "La participación de los padres en el proceso de enseñanzaaprendizaje de los hijos”, Tesis de Doctorado, Universidad de Concepción, Chile, en cooperación con la Universidad de Estocolmo, Suecia.

OIT (Organización Internacional de Trabajo sobre Pueblos Indígenas y Tribales en Países Independientes) (1989), Convenio 169 de la OIT sobre Pueblos Indigenas y Tribales en Paises Independientes, Chile: Mineduc.

Ouellet, Fernand (2002), "L'éducation interculturelle et l'éducation à la citoyenneté. Quelques pistes pour s'orienter dans la diversité des conception”, en VEI Enjeux, núm. 129, Francia: ACEPP Rhone Alpes.

Peralta, María (2012), El desarrollo de programas para la atención de la primera infancia de comunidades indigenas en Latinoamérica: algunas lecciones aprendidas, Chile: Editorial Universidad Central.

Peralta, María (2000), "Una propuesta de criterios de calidad para una educación inicial latinoamericana”, en Peralta, María y Salazar, R. [coords.], Calidad y modalidades alternativas en educación inicial, Bolivia: CERID/MAYSAL.

Quilaqueo, Daniel (2006), "Valores educativos mapuches para la formación de personas desde el discurso de kimches”, en Estudios Pedagógicos, núm. 2, Chile: Universidad Austral de Chile.

Quilaqueo, Daniel et al. (2014), "Saberes educativos mapuches: aportes epistémicos para un enfoque de educación intercultural”, en Revista Chungará, núm. 2, Chile: Universidad de Tarapacá.

Quintriqueo, Segundo (2010), Implicancias de un modelo curricular monocultural en contexto mapuche, Chile: LOM.

Quintriqueo, Segundo et al. (2014), "Formación del profesorado en Educación Intercultural en América Latina. El caso de Chile", en Revista Electrónica Interuniversitaria de Formación del Profesorado, núm. 2, España: AUFOP.

Quintriqueo, Segundo et al. (2015), "Relación entre el conocimiento educativo mapuche y el escolar: base para un currículum intercultural”, en Quilaqueo, Daniel, Quintriqueo, Segundo y Peña, Fernando [coords.], Interculturalidad en contexto de diversidad social y cultural: Desafios de la Investigación Educativa en Contexto Indigena, Chile: Editorial Universidad Católica de Temuco.

Quintriqueo, Segundo et al. (2016), Interculturalidad en la formación inicial docente: invitación a construir un dialogo intercultural, Chile: Universidad Católica de Temuco.

Ramírez, Ángel (2001), "Paradigma de la interculturalidad", en Publicación mensual del Instituto Cientifico de Culturas Indígenas, núm. 26. Disponible en: http://icci. nativeweb.org/boletin/26/ramirez.html [10 de enero de 2017]. 
Soledad Morales-Saavedra, Segundo Quintriqueo-Millán, Pilar Andrea Uribe-Sepúlveda y Katerin Arias-Oretga. Interculturalidad en educación superior: experiencia en educación inicial en La Araucania, Chile

Santelices, Lucía (2002), "La colaboración entre la familia y la escuela: un factor para mejorar la calidad de la educación”, en Revista de Pedagogía, núm. 426, España: REP.

Schmelkes, Sylvia (2009), “Interculturalidad, democracia y formación valoral en México”, en Revista Electrónica de Investigación Educativa, núm. 2, México: Universidad Autónoma de Baja California.

Schmelkes, Sylvia (2012), "Educación para un México intercultural”, en Revista Sinéctica, núm. 40, México: Universidad Jesuita de Guadalajara.

Schon, Donald (1992), La formación de profesionales reflexivos. Hacia un nuevo diseño de la enseñanza y aprendizaje de las profesiones, España: Paidós.

Tubino, Fidel (2011), "El nivel epistémico de los conflictos interculturales", en Revista Construyendo Nuestra Interculturalidad, núm. 7, Perú: doi.org/10.17141/ iconos.60.2018.2922

Tubino, Fidel (2014), "El trasfondo epistémico de los conflictos interculturales", en Revista Contexto Latinoamericano, núm. 1, Brasil: Ocean Sur.

Tubino, Fidel (2005b), "La interculturalidad crítica como proyecto ético-político", en Encuentro continental de educadores agustinos. Disponible en: http://oala.villanova. edu/congresos/educación/lima-ponen-02.html. [10 de enero de 2017].

Tubino, Fidel y Ardito, Wilfredo (1996), Interculturalidad: un desafío, Perú: Centro Amazónico de Antropología y Aplicación Práctica.

Valverde, Paz (2008), "Hacia una Práctica Docente de Calidad en el Trabajo con Familia: Articulando Expectativas de Instituciones Formadoras, Política Educativa y las Familias de la Educación Inicial", en Proyecto Fondo de Investigación y Desarrollo en Educación, núm. F420932-2009, Chile: Fondecyt.

Valdivia, Marcela y Valverde, Paz (2006), "La participación de los padres en los jardines infantiles", en Boletín de Investigación Educacional, núm. 2, Chile: Pontificia Universidad Católica de Chile.

Veenman, Simon (1984), "Perceived problems of beginning teachers", en Review of Educational Research, núm. 2, Inglaterra: Review of Educational Research.

Viaña, Jorge et al. (2010), Construyendo Interculturalidad Critica, Ecuador: Instituto Internacional de Integración del Convenio Andrés Bello.

Soledad Morales-Saavedra. Magíster Psicología, Profesora Asociada de la Universidad Católica de Temuco. Investigadora adjunta del Centro de Investigación en Educación en Contexto Indígena e Intercultural (CIECII). Línea de investigación: educación e interculturalidad. Publicación reciente: en coautoría con S. Quintriqueo, D. Quilaqueo y K. Arias, Interculturalidad para la Formación Inicial Docente: Desafios para construir un diálogo intercultural, Chile: Editorial Universidad Católica de Temuco (2016).

Segundo Quintriqueo-Millán. Doctor en Educación, profesor asociado de la Universidad Católica de Temuco, Chile; investigador asociado del Centro de Investigación en Educación en Contexto Indígena e Intercultural (CIECII); investigador del Núcleo de Estudios Interétnicos e Interculturales (NEII) de 
la UC Temuco e investigador asociado del Centre Interuniversitaired'Études et de Recherches Autochtones (CIÉRA) de l'Université Laval, Québec, Canadá. Línea de investigación: educación e interculturalidad. Publicaciones recientes: en autoría con S. Quintriqueo, D. Quilaqueo, F. Peña-Cortés y G. Muñoz, "Conocimientos culturales como contenidos de la educación familiar mapuche”, en Revista ALPHA, núm. 40, Chile (2015); en autoría con S. Quintriqueo, D. Quilaqueo y H. Torres, "Contribución para la enseñanza de las ciencias naturales: saber mapuche y escolar", en Revista Educacion y Pesquisa, vol. 40, núm. 4, Brasil (2014).

Pilar Andrea Uribe-Sepúlveda. Doctora en Investigación Educativa, Académica de la Carrera Educación Parvularia. Línea de investigación: educación e interculturalidad. Publicación reciente: Cuadrado-Gordillo, Isabel, Uribe-Sepúlveda, Pilar A. y Cadet, Bernard, "The Case of the Mapuche People in Chile", en Giraudeau, Caroline [coord.], Psychologie, éducation et vie scolaire, Francia: Publibook Université.

Katerin Arias-Ortega. Estudiante de Doctorado en Educación, Universidad Católica de Temuco, Chile. Línea de investigación: educación e interculturalidad. Publicación reciente: en coautoría con S. Quintriqueo, S. Morales, D. Quilaqueo y K. Arias, Interculturalidad para la Formación Inicial Docente: Desafíos para construir un diálogo intercultural, Chile: Editorial Universidad Católica de Temuco (2016).

Recepción: 20 de septiembre de 2017.

Aprobación: 15 de febrero de 2018. 